\title{
Dual-Sensor Package Technology Supporting ASIL-D in Safety-Related Automotive Applications
}

\author{
Wolfgang Scheibenzuber ${ }^{1}$, Franziska Moissf', Julao Nimler Elemparo ${ }^{3}$, Ovaldo Borghetti', \\ Harald Witschnig ${ }^{4}$, Friedrich Rasbornig ${ }^{4}$ \\ ${ }^{1}$ Infineon Technologies AG, Am Campeon 1-12, 85579 Neubiberg, Germany, \\ Wolfgang.Scheibenzuber@Infineon.com, \\ ${ }^{2}$ Infineon Technologies AG, Wernerwerkstrasse 2, 93049 Regensburg, Germany \\ ${ }^{3}$ Infineon Technologies Malaysia Sdn. Bhd., Lot 10 \& 11 Kulim Hi-Tech Park, 9000 Kulim, Malaysia \\ ${ }^{4}$ Infineon Technologies Austria AG, Siemensstraße 2, 9500 Villach, Austria
}

\begin{abstract}
:
We present a dual-sensor package technology allowing integration of two separate sensor chips in just one device, which has the same dimensions as a regular single-chip sensor. By offering built-in redundancy, the dual-sensor package supports the achievement of the highest safety level, ASIL-D in safety-related automotive applications.

The chips are placed on top and bottom of the package leadframe, so that the lateral position of the sensing elements coincides. This mounting technique ensures a maximum relative measurement accuracy of the two sensor chips. Furthermore, the small footprint of the dual-sensor package enables the implementation of redundant sensors in applications with limited space, thus saving precious space and cost.
\end{abstract}

Key words: Functional Safety, Dual-Sensor, Position Sensor, EPS, Electric Power Steering

\section{Achieving ASIL-D in Safety-Related Applications}

In safety-related automotive applications, such as electric power steering (EPS), the ISO 26262 standard [1] sets high requirements for the diagnostic coverage of random failures and the avoidance of systematic failures to reach the highest safety level ASIL-D. For ASIL-D rated position sensor applications, these high requirements can typically be achieved by using redundant sensors and comparing their signals on a microcontroller.

Infineon's PG-TDSO dual-sensor package integrates two linear Hall sensors or two angle sensors. Both sensors have separate power supply and separate signal outputs. They are electrically independent due to galvanic isolation, and have the same footprint as conventional single sensor devices. This means that the two sensors work independently which increases reliability of the system.

\section{Dual-Sensors for Electric Power Steering Applications (EPS)}

Dual-die position sensors are commonly used in safety-related automotive applications. In an EPS system (see Fig. 1), there are several position sensing applications which are rated with the highest functional safety level, ASIL D.
The input signal for the steering assistance is given by a steering torque sensor that translates the torque exerted on the steering column by the driver via a torsion bar and a suitable magnetic circuit into a magnetic signal. This magnetic signal is then measured by a linear Hall effect magnetic position sensor. To prevent potentially hazardous events, such as self-steering or erroneous steering support, the highest level of safety integrity is required for the torque sensor signal. This is typically accomplished by using the signals of two redundant sensors. Premium EPS systems use even more redundant torque sensors to increase the availability of the system. Infineon provides the highly accurate TLE499xx8D series of linear Hall sensors as dual-sensor package with a variety of communication interfaces to fit different system architectures.

A typical electric motor that provides the steering support in an EPS system has a rotary magnetic position sensor for commutation. In application like this, incorrect commutation of the motor may cause potentially hazardous events. For this reason, it is necessary that the position sensor also fulfills ASIL-D safety requirements. The TLE5012BD high performance GMR (Giant-Magneto-Resistance) digital angle sensor or, depending on module architecture, also the fast analog TLE5x09A16D 


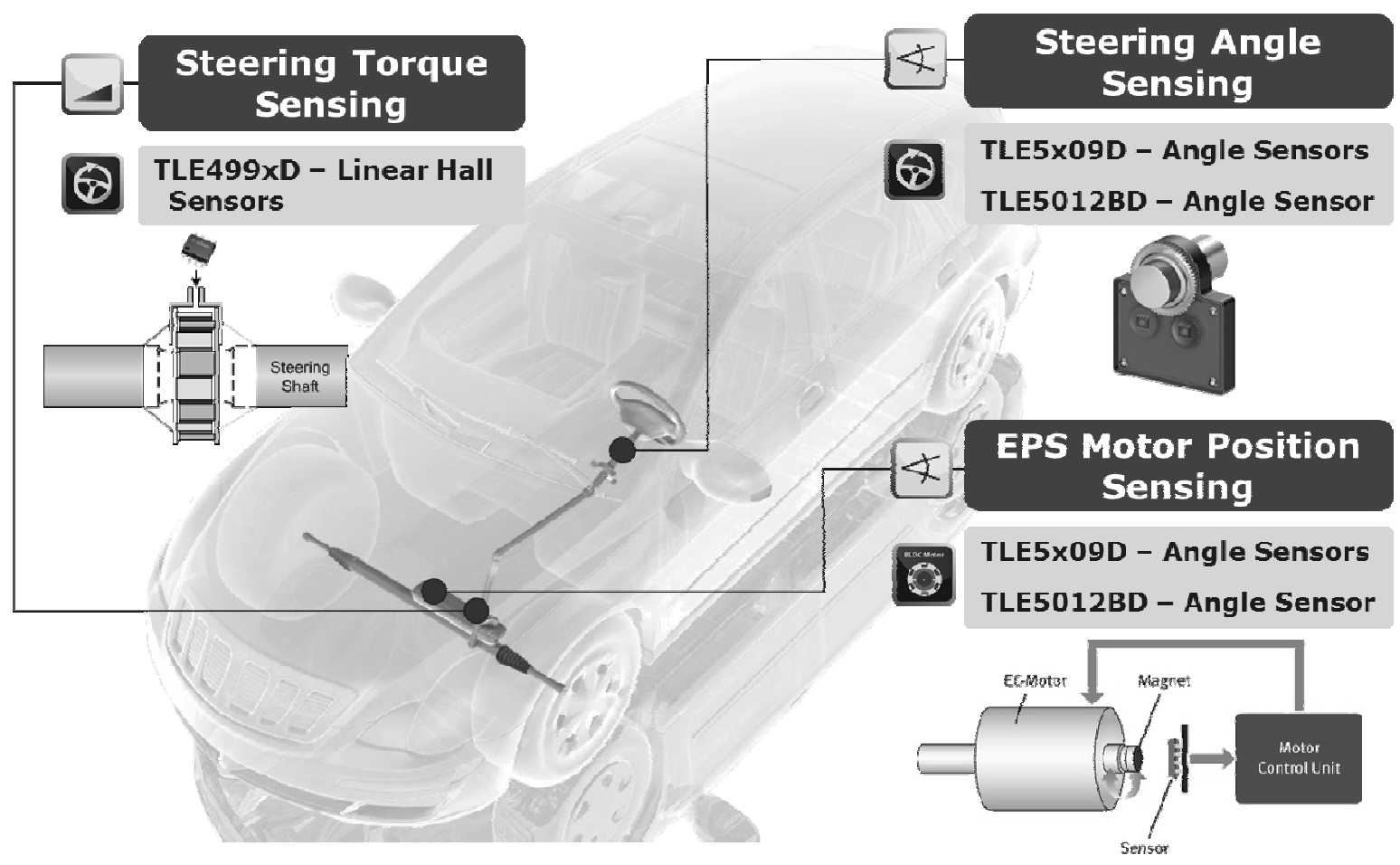

Fig. 1: Safety-relevant applications for redundant position sensors in an electric power steering system.

series in the dual-sensor package are the ideal solution for applications like this.

A further magnetic position sensor application in the EPS system is steering angle sensing. The steering angle is an input signal for vehicle stability assistant systems, such as the electronic stability program (ESP). In this application, typically a Vernier configuration of two magnetic angle sensors on cogwheels is used (see Fig. 1). This configuration can be sufficient for achieving ASIL-D rating in operation, but is not during the vehicle's starting phase. Premium EPS systems in addition, require ASIL-D from start, which can be fulfilled by using redundant dual-sensors, like TLE5012BD, for each cogwheel.

\section{Bus Capability with SPC Interface}

With conventional sensors that use analog signals or digital PWM or SENT interfaces, the implementation of additional sensing channels in a system to account for increased safety and reliability requirements typically adds considerable cost due to the additional wiring that is necessary to connect all the sensors. These costs can be saved by using the ShortPWM-Code (SPC) interface [2], which is available for the linear Hall and magnetoresistive angle sensors in the dual sensor package. The SPC interface allows the combination of up to four sensors on one digital line and thus reduces the total number of wires necessary for a system. Furthermore, it also allows connecting sensors of different types, which enables additional cost saving in combined modules, like torque-and-angle sensors (TAS) [3].

\section{Redundancy and Diversity}

The flexibility of the dual-sensor package, offers on the one hand two identical sensor chips in one package, but also the possibility to combine sensor chips with different sensing principles such as the TLE5309D, which uses an AMR [4] and a GMR [5] magnetic angle sensor. This leads to functional enhancement and a sensing solution that is redundant and diverse in one

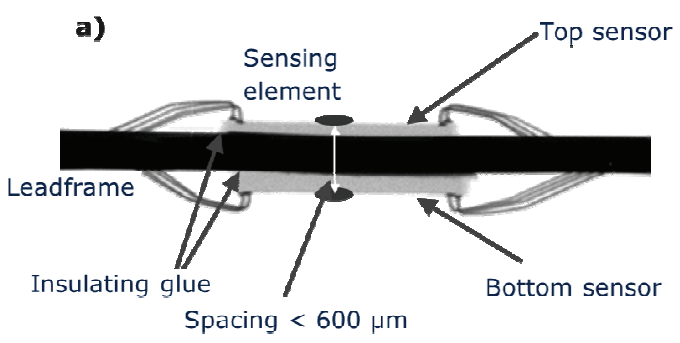

b)

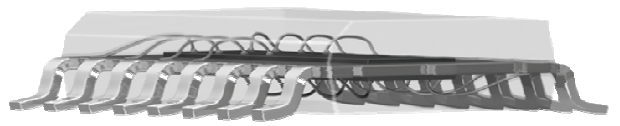

Fig. 2: a) Side view of stack-mounted dual sensors with bonding wires; b) transparent 3Dgraph of dual-sensor PG-TDSO-16-2 package 
package. This integrated diversity ensures that the highest requirements for functional safety are fulfilled.

\section{Stack Mounting}

By using an innovative stack mounting technology (see Fig. 2), two independent magnetic position sensors are integrated in space-saving PG-TDSO packages only about 1 $\mathrm{mm}$ thin, but with the same width and length as a conventional single sensor package. Compared to the conventional approach of placing two sensors side-by-side, the topbottom placement (see Fig. 3) offers several advantages:

The spatial separation between the two sensitive elements is considerably smaller, typically less than $600 \mu \mathrm{m}$. Additionally, the lateral position of the sensitive elements inside the package coincides. This greatly improves the matching of the two sensors' signals and thus also the capability for plausibility comparison and the overall performance on system level.

Furthermore this matching improvement leads to cost saving on system level, as expensive rare earth magnets that ensure a wide spatial

a) Infineon dual sensor package solution: face up/ face down assembly technology

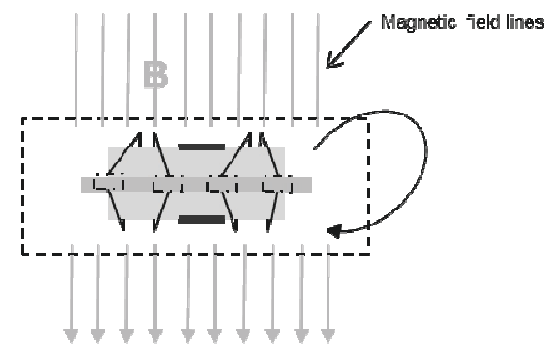

b) Conventional dual sensor solution: redundant sensor chip by chip

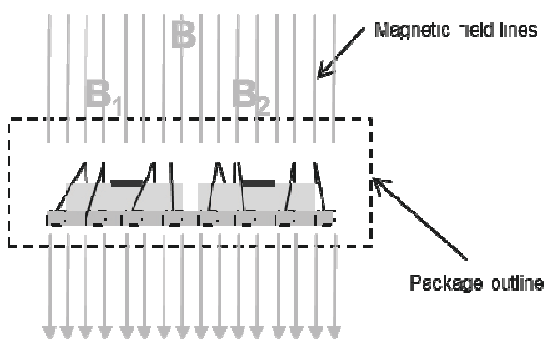

Fig. 3: a) Top-Bottom dual-sensor package solution: smaller footprint and more homogeneous magnetic field at the sensing elements;

b) Conventional dual-sensor approach: large footprint and inhomogeneous field distribution. homogeneity of the magnetic field, are no longer required. Instead, lower cost ferrite magnets can be used.

A further advantage of the dual-sensor package is its small footprint, being the same as for a typical single sensor package. This is of particular benefit in application environments where space is very limited due to the geometry of the magnetic circuitry i.e. steering torque sensing. Here, the dual-sensor package allows putting two sensor chips in the place of one.

\section{Summary}

As conclusion, the dual-sensor package supports fulfillment of the strict ISO 26262 requirements by providing integrated redundancy and diversity. Highly accurate analog and digital linear Hall sensors and magneto-resistive angle sensors are available as dual-sensor package for various safetyrelated automotive position sensing applications. Owing to the stack mounting technology, the dual-sensor package additionally allows cost saving on system level and an improved performance compared to conventional side-by-side dual-sensor configurations.

\section{References}

[1] ISO 26262 Road vehicles - Functional safety. International Standard, 2011

[2] Beaurenaut, L., Eggiman, C., Gastinger, F., Platzdasch, $\mathrm{H}$. et al., "Single-Edge Nibble Transmission: Challenges and Evolutions," SAE Technical Paper 2009-01-0125, 2009, doi:10.4271/2009-01-0125.

[3] Yoo, K., Seo, J., Ban, J., Hyukjung, L. et al., "Study on Development of Torque and Angle Sensor for EPS," SAE Int. J. Passeng. Cars Electron. Electr. Syst. 5(1):292-296, 2012, doi:10.4271/2012-01-0941.

[4] Witschnig H., Morici A., Schaffer B., Zimmer J. (2013). A Fully Monolithic Integrated Anisotropic Magnetoresistance Based Angle Sensor For Automotive. In Proc. 17th International Conference on Solid-State Sensors, Actuators and Microsystems TRANSDUCERS 2013 (EUROSENSORS XXVII). Barcelona Spain, 16 20 June 2013. pp. 2257-2260. ISBN: 978-1-46735983-2.

[5] Granig W. , Kolle C. , Hammerschmidt D., Schaffer B. , Borgschulze R., Reidl C., and Zimmer J., "Integrated giant magnetic resistance based angle sensor," in 5th IEEE Conference on Sensors, 2006, October 2006, pp.542-545. 